\title{
2016-Nag Rios-AAC-tn27372
}

Suitably equipped global and local air traffic can be tracked. The tracking information may then beused for control from ground-based stations by receiving the Automatic Dependent Surveillance-Broadcast (ADSB) signal. The ADS-B signal, emitted from the aircraft's Mode-S transponder, is currently tracked by terrestrial based receivers but not over remote oceans or sparsely populated regions such as Alaska or the Pacific Ocean. Lack of real-time aircraft time/location information in remote areas significantly hindersoptimal planning and control because bigger "safety bubbles" (lateral and vertical separation) are required around the aircraftuntil they reach radar-controlled airspace. Moreover, it presents a search-andrescue bottleneck. Aircraftin distress, e.g. Air France AF449 that crashed in 2009, take days to be located or cannot be located at all, e.g. Malaysia Airlines MH370 in 2014. In this paper, we describea tool for designing a constellation of small satellites which demonstrates, through high-fidelity modelingbased on simulatedair traffic data, the value of space-based ADS-B monitoringand provides recommendations forcost-efficient deployment of a constellation of small satellites to increase safety and situational awareness inthecurrently poorly-served surveillance area of Alaska.Air traffic data has been obtained from the Future ATM Concepts Evaluation Tool (FACET), developed atNASA Ames Research Center, simulated over the Alaskan airspace over a period of one day. The simulation is driven by MATLAB withsatellites propagated and coverage calculated using AGI's Satellite ToolKit(STK10). 